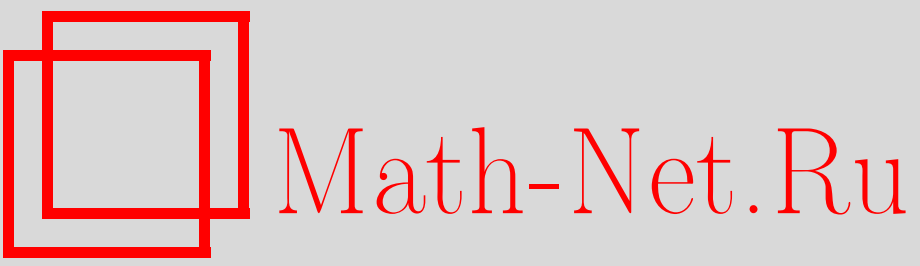

Ю. А. Кашлев, Н. М. Садыков, Неравновесная статистическая термодинамика каналированных частиц. Резонансные переходы и эффект деканалирования, TMФ, 1998, том 116, номер 1, 146-160

DOI: https://doi.org/10.4213/tmf894

Использование Общероссийского математического портала Math-Net.Ru подразумевает, что вы прочитали и согласны с пользовательским соглашением

http://www.mathnet.ru/rus/agreement

Параметры загрузки:

IP: 44.207 .124 .84

26 апреля 2023 г., $13: 27: 48$ 


\author{
ТЕОРЕТИЧЕСКАЯ \\ И МАТЕМАТИЧЕСКАЯ \\ ФИЗИКА \\ Том 116, № 1 \\ июль, 1998
}

(C) $\mathbf{1 9 9 8}$ г.

Ю. А. Кашлев ${ }^{*}$, Н.М. Садыков ${ }^{\dagger}$

\title{
НЕРАВНОВЕСНАЯ СТАТИСТИЧЕСКАЯ ТЕРМОДИНАМИКА КАНАЛИРОВАННЫХ ЧАСТИЦ. РЕЗОНАНСНЫЕ ПЕРЕХОДЫ И ЭФФЕКТ ДЕКАНАЛИРОВАНИЯ
}

Утверждается, что элементарный акт деканалирования включает в себя диффузию частиц в пространстве поперечных энергий и (после достижения частицей уровня, соответствующего потенциалу захвата в режим каналирования) резонансный переход. Определение коэффициента скорости деканалирования дано в основе уравнений неравновесной статистической термодинамики. Физические величины, вошедшие в коэффициент деканалирования, выражены через основные параметры теории, а именно, получены матричный элемент резонансного перехода, релаксационные постоянные в условиях однофононного и электронного рассеяний, а также ффункция поперечной квазитемпературы частиц, обусловленная разрывом термодинамических параметров быстрых частиц и термостата.

\section{1. ВВЕДЕНИЕ}

Согласно нелокальной классической теории Линдхарда [1] при углах падения пучка (относительно кристаллографической плоскости), меньших критического, распределение быстрых частиц, движущихся в кристалле, разделяется на две части: каналированную (направленную) и хаотическую. Каналированные частицы (КЧ) движутся в центре канала, совершая периодические отражения от кристаллографических плоскостей, образуюших стенки канала. Что же касается частиц хаотической части пучка, то они движутся по прямым траекториям под произвольными углами к плоскости канала. В условиях коррелированных столкновений воздействие регулярной “жесткой” решетки на КЧ описывается с помощью эффективного периодического потенциала $U_{\text {eff }}(x)$ с величиной потенциального барьера $U_{0}$. Предполагается, что плоскость канала параллельна плоскости $(y, z)$, а поперечное движение КЧ совершается в $x$-направлении.

В локальной классической теории [2-4] было показано, что возможен еще один режим направленного движения частиц - квазиканалирование. В этом режиме частица

\footnotetext{
* Институт металлургии им. А. А. Байкова РАН, Москва, Россия

${ }^{\dagger}$ Актауский университет им. ШІ. Е. Есенова, Актауск, Республика Казахстан
} 


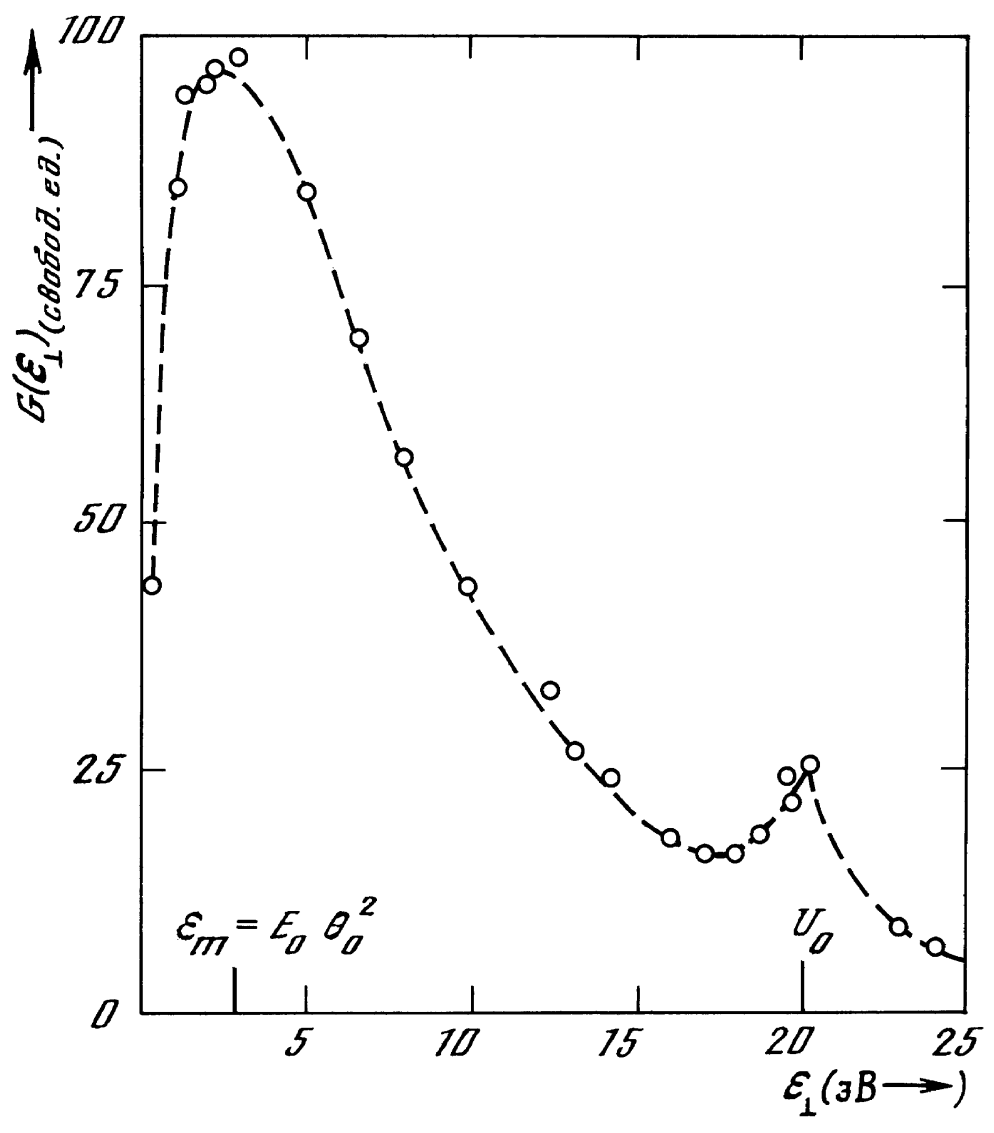

Рис. 1

движется вдоль стенки канала в непосредственной близости от нее. При этом она может совершать случайным образом переходы от одной стенки к соседней, продолжая затем направленное движение вдоль последней. Локальная теория установила различие вкладов, которые дают частицы, движушиеся в режимах каналирования, квазиканалирования и хаотического движения, как в равновесные, так и в кинетические физические величины. Рассмотрим в качестве примера плотность числа состояний направленного пучка частищ $G\left(\varepsilon_{\perp}\right)$ как функцию поперечной энергии частиц $\varepsilon_{\perp}$. Локальное уравнение (см. приложение), как показано на рис. 1, дает немонотонную функцию. Наличие максимума у $G\left(\varepsilon_{\perp}\right)$ в области малых значений поперечной энергии связано с тем, что ограничение заданным углом влета в канал $\theta_{0}$ приводит к заполнению (на глубине порядка длины когерентности) узкого интервала поперечных энергий вблизи энергии влета $\varepsilon_{m}=E_{0} \theta_{0}^{2}$. Здесь $E_{0}$ - начальная энергия частицы. Вместе с тем на рис. 1 имеется еше и меньший по высоте максимум. Последний соответствует частицам, движушимся в режиме квазиканалирования, и поскольку зона (или уровень) квазиканалирования энергетически примыкает к вершине потенциального барьера, второй максимум 
$G\left(\varepsilon_{\perp}\right)$ соответствует значению $\varepsilon_{\perp}=U_{0}$. Отметим, что экспериментальное подтверждение эффекта квазиканалирования (на рис. 1 обозначено кружочками) обсуждалось в работах [5-9].

В квантовой модели каналирования движение частицы направленного пучка в плоскости канала считается свободным, а соответствуюшая энергия - непрерывной. В то же время энергия поперечного движения дискретна. Чтобы дать представление о квантовых состояниях частиц, достаточно ввести два модельных потенциала:

$$
\begin{aligned}
U_{2}\left(x^{\prime}\right) & =\frac{m}{2}\left(\Omega Q\left(x^{\prime}\right)\right)^{2}, \\
U_{3}\left(x^{\prime \prime}\right) & =\frac{m}{2}\left(\Omega Q\left(x^{\prime \prime}\right)\right)^{2}+\omega_{s_{0}} .
\end{aligned}
$$

Здесь $U_{2}\left(x^{\prime}\right)$ и $U_{3}\left(x^{\prime \prime}\right)$ - соответственно потенциалы КЧ и частицы, движущейся в режиме квазиканалирования, $m$ - масса частицы, $Q\left(x^{\prime}\right)$ - поперечное смешение из положения равновесия $x^{\prime}$,

$$
\Omega=\frac{\pi}{l}\left(\frac{\omega_{s_{0}}}{m}\right)^{1 / 2}
$$

- частота поперечных колебаний, $\omega_{s_{0}}$ - потенциал захвата частицы в режим каналирования [10], которому в классической теории соответствует барьер $U_{0}, l$ - полуширина канала. Если минимум $U_{2}\left(x^{\prime}\right)$ находится в середине канала $x^{\prime}$, то минимум $U_{3}\left(x^{\prime \prime}\right)-$ в непосредственной окрестности атомной плоскости, образующей стенку канала. При этом в плоскостном потенциале $U_{2}\left(x^{\prime}\right)$ имеется большое число энергетических уровней $\omega_{s}=\Omega s^{1)}$, где $s-$ колебательное квантовое число. Чтобы описать состояние КЧ, достаточно задать числа заполнения $n_{s}^{0}$ квантовых состояний $s$. Числа $n_{s}^{0}$ будем считать собственными значениями оператора $\stackrel{+}{a}_{s} a_{s}$, где операторы $\stackrel{+}{a}$ и $a_{s}$ удовлетворяют перестановочным соотношениям Ферми $[11,12]$. Что же касается состояний квазиканалированной частицы, то из всех состояний в яме $U_{3}\left(x^{\prime \prime}\right)$ в дальнейшем ограничимся рассмотрением ее основного состояния с энергией $E_{3}^{(0)}$. Волновая функция основного состояния имеет максимум вблизи стенки канала [13]. Для более подробного описания возбужденных состояний ямы $U_{3}\left(x^{\prime \prime}\right)$ можно ввести зоны конечной ширины, причем по мере увеличения номера зоны движение частицы приближается к свободному [13], т.е. к движению частицы хаотической части пучка.

Итак, рассматриваемая нами система разделяется на три части: 1) термостат $\{i=$ $1\}$, включающий решетку и электронный газ; 2) хаотическую часть пучка; 3) направленный пучок частиц, который имеет две составляющие, а именно $\mathrm{K} Ч\{i=2\}$, находяшиеся в состояниях $s$ в многоуровневых ямах $U_{2}\left(x^{\prime}\right)$, и квазиканалированные частицы $\{i=3\}$, находящиеся в основном состоянии потенциальных ям $U_{3}\left(x^{\prime \prime}\right)$.

\footnotetext{
1) Принята система единиц, в которой $\hbar=k_{\mathrm{B}}=1$.
} 


\section{2. КОЭФФИЦИЕНТ СКОРОСТИ ДИФФУЗИОННОГО ДЕКАНАЛИРОВАНИЯ И РЕЗОНАНСНЫЙ ПЕРЕХОД}

Поскольку квазиканалированные частишы быстро у ходят в хаотическую часть пучка [5], из двух составляюших направленного пучка короткоживущими считаются состояния подсистемы $\{3\}$. Поэтому при изучении деканалирования, т.е. выхода КЧ из режима каналирования, достаточно учесть [11] скорость перехода $\{2\} \rightarrow\{3\}$. В общем виде скорость такого перехода была получена в статье [11] на основе анализа обобщенных уравнений переноса числа частищ и энергии в условиях каналирования.

Найдем коэффишиент скорости диффузионного деканалирования. Запишем обший вид коэффициента скорости [11]:

$$
R=\frac{\pi}{4}\left|J_{23}\right|^{2} \int_{-\infty}^{\infty} d \omega I(-\omega) K_{1}(\omega)
$$

Здесь $J_{23}$ - матричный элемент перехода $\{2\} \rightarrow\{3\}, I(\omega)$ - спектральная интенсивность корреляционной функции плотность-плотность

$$
\begin{aligned}
& \left\langle\rho(X) \rho\left(X, t^{\prime}\right)\right\rangle_{l}=\int_{-\infty}^{\infty} d \omega I(\omega ; X, X) \exp \left(-i \omega t^{\prime}\right), \\
& \rho(X)=\sum_{s, s^{\prime} \leqslant s_{0}}\left\langle s|n| s^{\prime}\right\rangle \stackrel{+}{a}_{s} a_{s^{\prime}} \exp \left\{-i \frac{\pi}{l}\left(s-s^{\prime}\right) X\right\}
\end{aligned}
$$

- плотность числа КЧ в яме с координатой $X,\left\langle s|n| s^{\prime}\right\rangle$ - матричный элемент оператора плотности. В случае малой плотности КЧ, когда корреляция между координатами заполненных ям фактически отсутствует, интенсивность $I(\omega ; X, X)$ не зависит от хаотических переменных $X$, так что $I(\omega ; X, X)=I(\omega)$. Функция $K_{1}$ из (1) является спектральной интенсивностью корреляционной функции от переменных внешних степеней свободы. Действительно, частица направленного пучка находится либо в одном из состояний многоуровневой ямы $U_{2}$, либо в основном состоянии ямы $U_{3}$ с энергией $E_{3}^{(0)}$. Если для описания этой альтернативы ввести оператор $\stackrel{+}{c} c$ числа заполнения состояния ямы $U_{2}$ и оператор $\stackrel{+}{d} d$ числа заполнения состояния $U_{3}$, то имеем

$$
\int_{-\infty}^{\infty} d \omega \exp (-i \omega t) K_{1}(\omega)=\left\langle\stackrel{+}{c} \exp \left(i H^{(0)} t\right) d_{c}^{+} \exp \left(-i H^{(0)} t\right)\right\rangle_{l}^{(0)} .
$$

Спектральную интенсивность $K_{1}(\omega)$ достаточно вычислить, пренебрегая взаимодействием, поэтому в соотношение (3) входит гамильтониан невзаимодействуюших частиц $H^{(0)}$.

Если направленный пучок частиц рассматривать как самостоятельную термодинамическую систему, то для ее описания достаточно использовать гамильтониан $H_{i}$, число частиц $N_{i}$, а также два сопряженных этим величинам термодинамических параметра $F_{i 1}$ и $F_{i 2}$. При таком описании усреднение $\langle\ldots\rangle_{l}$ в $(2 \mathrm{a}),(3)$ выполняется с квазиравновесным статистическим оператором вида

$$
\rho_{l}=\frac{1}{Q_{l}} \exp \left\{-\sum_{i=2,3}\left(F_{i 1} H_{i}+F_{i 2} N_{i}\right)\right\},
$$


где $Q_{l}$ - статистический функционал. Основной термодинамический параметр - поперечная квазитемпература КЧ $F_{21}^{-1}$ устанавливается за счет конкуренции двух процессов: диффузии частиц в пространстве поперечной энергии и диссипативного процесса [14]. Медленность передачи поперечной энергии частиц приводит к значительному "разогреву" направленного пучка. Оценки показали [14], что в случае $E_{0} \sim 1 \mathrm{MэB} \mathrm{зна-}$ чения $F_{21}^{-1}$ лежат в интервале $(1-10)$ эВ, так что выполняется условие

$$
F_{21}=F_{31} \ll F_{11} \equiv \beta_{1},
$$

где $\beta_{1}^{-1}$ - температура термостата. Следовательно, система в условиях каналирования находится в состоянии, значительно удаленном от термодинамического равновесия.

Используя соотношение (2), нетрудно связать корреляционную функцию

$$
\left\langle\rho(X) \rho\left(X, t^{\prime}\right)\right\rangle_{l}
$$

с двухвременной запаздываюшей функцией Грина

$$
G_{s s^{\prime} m^{\prime} m}(t)=-i \Theta(t)\left\langle\left[\stackrel{+}{a}_{s}(t) a_{s^{\prime}}(t), \stackrel{+}{a}_{m^{\prime}} a_{m}\right]\right\rangle_{l}
$$

где $[A, B]=A B+B A, \Theta(x)$ - тета-функция. Функция Грина (4) удовлетворяет конечно-разностному уравнению типа уравнения Фоккера-Планка [12], которое одновременно описывает когерентный характер движения КЧ в регулярном кристалле и неупругие процессы рассеяния. Анализ этого уравнения показал [12], что оно имеет аналитическое решение в классе вырожденных гипергеометрических функций

$$
G_{s s^{\prime} m^{\prime} m}(t)=G_{s s^{\prime} m^{\prime} m}^{(0)}(t) \frac{1}{n_{s}+n_{s^{\prime}}} \sum_{s^{\prime \prime}} B_{s^{\prime \prime} 1} F_{1}\left(-s^{\prime \prime}, 1 ; r^{\prime}\right) \exp \left(-\gamma_{s^{\prime \prime}} t\right),
$$

где $G_{s s^{\prime} m^{\prime} m}^{(0)}(t)$ - функция Грина нулевого порядка по взаимодействию. Используя это решение, коэффициент (1) запишем в виде

$$
\begin{aligned}
R= & \frac{1}{4}\left|J_{23}\right|^{2} \int d \omega K_{1}(\omega) \frac{1}{\exp \left(-F_{21} \omega\right)+1} \times \\
& \times \sum_{s, s^{\prime}} \sum_{s^{\prime \prime}} B_{s^{\prime \prime}}\left|\left\langle s|n| s^{\prime}\right\rangle\right|^{2} \frac{\gamma_{s^{\prime \prime}}}{\left(\omega-\omega_{s s^{\prime}}\right)^{2}+\gamma_{s^{\prime \prime}}^{2}}{ }_{1} F_{1}\left(-s^{\prime \prime}, 1 ; r^{\prime}\right),
\end{aligned}
$$

здесь $\omega_{s s^{\prime}}=\omega_{s}-\omega_{s^{\prime}}, \gamma_{s^{\prime \prime}}$ - затухание, которое выражается через мнимую часть массового оператора [12] $\operatorname{Im} M_{s^{\prime \prime}}(\omega \pm i \bar{\varepsilon})=\mp \gamma_{s^{\prime \prime}}(\omega)$, где $\bar{\varepsilon} \rightarrow+0$. Коэффициенты $B_{s^{\prime \prime}}$ из $(5)$, в свою очередь, удовлетворяют уравнению

$$
\sum_{s^{\prime \prime}} B_{s^{\prime \prime}} F_{1}\left(-s^{\prime \prime}, 1 ; r^{\prime}\right)=2 n_{s}
$$




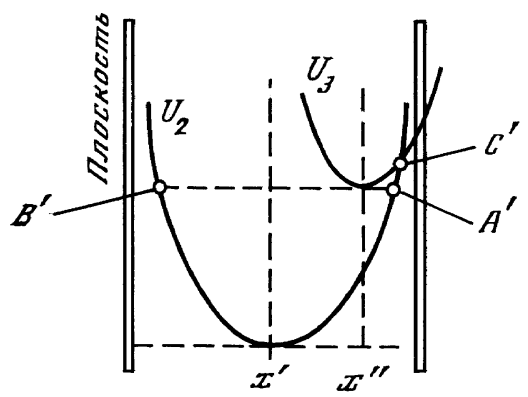

Рис. 2

где $n_{s}$ - функция распределения КЧ в состоянии квазиравновесия, т.е. на глубине проникновения порядка длины когерентности. Функция распределения нормирована, т.е.

$$
\sum_{s} n_{s}=1
$$

Переменная $r^{\prime}$, вошедшая в $(5),(6)$, положительна и связана с переменной $s$ трансцендентным уравнением

$$
\begin{aligned}
r^{\prime}\left\{{ }_{1} F_{1}\left(s, 1 ; r^{\prime}\right)+\Delta_{s r^{\prime}}\right\}= & s{ }_{1} F_{1}\left(s, 1 ; r^{\prime}\right)+\{1-\exp (-\xi)\} \times \\
& \times\left\{s_{1} F_{1}\left(s-1,1 ; r^{\prime}\right)-(s+1){ }_{1} F_{1}\left(s, 1 ; r^{\prime}\right)\right\},
\end{aligned}
$$

где $\Delta_{s r^{\prime}}={ }_{1} F_{1}\left(s+1,2 ; r^{\prime}\right)-{ }_{1} F_{1}\left(s, 2 ; r^{\prime}\right), \xi=\beta_{1} \Omega$.

Отбрасывая малые поправки, включающие поперечную квазитемпературу, спектральную интенсивность в формуле (3) можно записать в приближении малой плотности. Имеем $K_{1}(\omega)=\delta\left(\omega-\omega_{s_{0}}-\varepsilon_{m}\right)$. Поскольку энергия влета КЧ мала, вычислим коэффициент скорости (5) в предельном случае $\varepsilon_{m} \rightarrow 0$. Предполагая, что взаимодействие является слабым, $\gamma_{s}^{2} \ll \Omega^{2}$, коэффициент (5) с учетом явного вида $K_{1}$ представим в виде

$$
R=\left(2 \omega_{s_{0}}\right)^{-1}\left|J_{23}\right|^{2} \nu\left(F_{21}\right) \sum_{s^{\prime}, s^{\prime \prime}} B_{s^{\prime \prime}} \gamma_{s^{\prime \prime} 1} F_{1}\left(-s^{\prime \prime}, 1 ; r^{\prime}\right)
$$

где $\nu\left(F_{21}\right)=\left\{\exp \left(F_{21} \omega_{s_{0}}\right)+1\right\}^{-1}$.

Рассмотрим резонансный переход (резонанс по поперечной энергии). При наличии энергетической диаграммы (рис. 2) резонансный переход частицы из состояния $\{2\}$ в состояние $\{3\}$ происходит только в том случае, если в результате случайных блужданий между уровнями поперечной энергии ямы $U_{2}$ частица достигает уровня $\omega_{s_{0}}$, к которому энергетически примыкает $E_{3}^{(0)}$. Переход $\{2\} \rightarrow\{3\}$ происходит либо через точку $A^{\prime}$, либо через точку $B^{\prime}$. В отличие от квантово-механического перехода, в классической теории, где потенциальные барьеры непроницаемы, переход $\{2\} \rightarrow\{3\}$ происходит только через точку пересечения потенциалов $C^{\prime}$. 
Отмеченные черты переходов в квантовой и классической трактовках имеют достаточно обший характер, хотя впервые они были рассмотрены в некотором специальном случае $[15,16]$. Запишем явный вид матричного элемента перехода в форме $[15,16]$

$$
J_{23}=2(2 \pi \Omega) w,
$$

где

$$
w=\frac{1}{4}\left(B_{p}-2 B_{p-1}+B_{p-2}\right)
$$

- проницаемость барьера,

$$
B_{p}=\left(2 k_{c}\right)^{p} 2 \exp \left(-2 k_{c}\right), \quad k_{c}=\frac{\Omega}{4} m \Delta_{x}^{2},
$$

$p=1 / 2, \Delta_{x}=\left|x^{\prime}-x^{\prime \prime}\right|-$ смешение положения равновесия частицы подсистемы $\{3\}$ относительно положения равновесия КЧ. В (9а) введен дополнительный множитель 2 по сравнению с работами $[15,16]$, что связано с учетом двух вариантов переходя: в полной схеме потенциалов яма $U_{3}$ может быть расположена как справа от $U_{2}$, так и слева от нее. Поскольку в дальнейшем мы ограничимся изучением движения легких атомных частиц (протонов, $\alpha$-частиц) с энергией $E_{0} \sim 1 \mathrm{MэB}$, то согласно сделанным оценкам $k_{c} \gg 1$. Поэтому в (9б) достаточно сохранить только первый член.

\section{3. ЧАСТОТА РЕЛАКСАЦИИ}

Вычислим затухание $\gamma_{s}^{(\mathrm{ph})}=\left(1 / \tau^{(\mathrm{ph})}\right)\left(\omega_{s} / \omega_{s_{0}}\right)$, обусловленное рассеянием КЧ на фононах. Чтобы получить по возможности простое аналитическое выражение для частоты релаксашии поперечной энергии $1 / \tau^{(\mathrm{ph})}$, будем считать [12], что взаимодействие КЧ с атомами решетки, совершаюшими тепловые колебания, описывается модельным потенциалом гауссовского вида. Более реалистический потенциал межатомного взаимодействия, мольеровская аппроксимация потенциала Томаса-Ферми, был использован в работе [4], где рассматривалось движение КЧ в “замороженной” решетке. Однако из-за сложности потенциала расчет удалось выполнить только численными методами.

Вычислим частоту релаксации в условиях однофононного рассеяния, сопровождаемого переходами КЧ между ближайшими уровнями $s \rightarrow s \pm 1$.

Осцилляции КЧ между плоскостями канала сопровождаются процессами неупругого рассеяния (потенциал $\delta U(x)$ [12]), что приводит к переходам КЧ $s \rightarrow s \pm 1$. Вместе с тем движение в плоскости канала сопровождается упругими столкновениями КЧ (безразмерный потенциал $V(r)[12])$ с передачей импульса $\Delta \mathbf{p}=\mathbf{p}^{\prime}-\mathbf{p}\left(\mathbf{p}=\left(p_{y}, p_{z}\right)\right.$, $\left.|\mathbf{p}|=\left|\mathbf{p}^{\prime}\right|\right)$. Используя "золотое правило", вероятность перехода запишем в виде

$$
\begin{aligned}
& W\left(s, \tilde{\nu}_{q} ; \mathbf{p} \mid s-1, \tilde{\nu}_{q}+1 ; \mathbf{p}^{\prime}\right)= \\
& \quad=2 \pi\left|\left\langle s, \tilde{\nu}_{q}|\delta U| s-1, \tilde{\nu}_{q}+1\right\rangle\right|^{2}\left|\left\langle\mathbf{p}|V| \mathbf{p}^{\prime}\right\rangle\right|^{2} \delta\left(\omega_{s}-\omega_{s-1}-\omega_{q}\right) .
\end{aligned}
$$


Согласно работе [12] имеем

$$
\begin{gathered}
\left\langle s, \tilde{\nu}_{q}|\delta U| s-1, \tilde{\nu}_{q}+1\right\rangle=I_{q} s^{1 / 2}, \quad I_{q}=-i \varkappa_{0}\left(\tilde{N} M \omega_{q} m \Omega\right)^{-1 / 2} \sin (q l), \\
V(r)=\frac{\bar{d}}{4 a}\left\{1+\left(\frac{\bar{d}}{2 r}\right)^{2}\right\}^{-1 / 2} \exp \left\{-\left(\frac{r}{4 a}\right)^{2}\right\}, \quad \mathbf{r}=\left(r_{y}, r_{z}\right) .
\end{gathered}
$$

В (10) использованы следуюшие обозначения: $\tilde{\nu}_{q}$ - среднее число заполнения фононов, $\varkappa_{0}$ - константа связи КЧ с решеткой, $\tilde{N}$ - число атомов в $x$-цепочке, $M$ - масса атома решетки, $\bar{d}=(N 2 l)^{-1 / 2}, N-$ плотность атомов решетки, $a-$ эффективньй радиус экранирования ионно-атомного взаимодействия, $\omega_{q}$ - энергия фонона. Вместо рассеяния КЧ на независимых колебаниях отдельных атомов решетки [17] мы рассмотрим взаимодействие КЧ с колебательной модой $x$-цепочки. Если при этом ограничиться учетом смещений атомов цепочки в поперечном относительно нее направлении, то, как показывает анализ, дисперсионные соотношения принимают вид

$$
\omega_{q}^{2}=\omega_{0}^{2}+\widetilde{\omega}^{2} \sin ^{2}(q l), \quad \omega_{0}^{2}=\frac{\tilde{g}_{c}}{M}, \quad \widetilde{\omega}^{2}=\frac{4 g_{c}}{M} .
$$

При выводе (11) использована модель $x$-цепочки с двумя силовыми постоянными $g_{c}$ и $\tilde{g}_{c}[12]$, которая применима в случае движения КЧ вдоль плотно упакованной плоскости.

В соответствии с квантовой теорией переходов [18] полная вероятность выхода частицы из потенциального минимума через возбужденное состояние $s_{0}$ с точностью до постоянного множителя равна вероятности последнего “шага" $\left(s_{0}-1\right) \rightarrow s_{0}$. Учитьвая это общее положение, а также стандартное представление [18] частоты релаксации в форме интегрального преобразования от вероятности перехода (10), получаем, что

$$
\frac{1}{\tau^{(\mathrm{ph})}}=2 \pi \frac{\omega_{s_{0}}}{\Omega} \Psi_{1}\left(v_{0}\right) \sum_{q}\left|I_{q}\right|^{2} \varphi\left(\beta_{1} \omega_{q}\right) \delta\left(\omega_{s_{0}}-\omega_{s_{0}-1}-\omega_{q}\right),
$$

где

$$
\Psi_{1}\left(v_{0}\right)=\sum_{\mathbf{p}^{\prime}}\left|\left\langle\mathbf{p}|V| \mathbf{p}^{\prime}\right\rangle\right|^{2}, \quad e^{-\beta_{1} \omega_{q}} \varphi\left(\beta_{1} \omega_{q}\right)=\tilde{\nu}_{q},
$$

$v_{0}$ - скорость КЧ.

Выполнив в (12) интегрирование по $q$, с учетом (10б) и (11) имеем

$$
\frac{1}{\tau^{(\mathrm{ph})}}=\frac{\Omega}{\widetilde{\omega}} \mathcal{G} \frac{\widetilde{\omega}}{\Omega} \varphi\left(\beta_{1} \Omega\right) \frac{1}{\tau_{1}\left(v_{0}\right)},
$$

где

$$
\begin{gathered}
\frac{1}{\tau_{1}\left(v_{0}\right)}=\chi_{1} \Psi_{1}\left(v_{0}\right), \quad \chi_{1}=(2 \pi)^{-2}\left|\varkappa_{0}\right|^{2} \frac{l^{2}}{g_{c}}, \\
\mathcal{G}(x)=\Theta\left(1-x^{2}\right)\left\{\left(1+\frac{4 g_{c}}{\tilde{g}_{c}}\right)-\left(\frac{1}{x}\right)^{2}\right\}^{-1 / 2}
\end{gathered}
$$


Вычислим частоту релаксации в случае энергетических зон конечной ширины.

Поскольку потенциал $U_{\text {eff }}(x)$ имеет вид периодической последовательности ям конечной высоты, аппроксимируем энергетический спектр КЧ известным выражением $E_{s}\left(\tilde{k}_{x}\right)=E_{s}(0)+\Delta \omega_{s} f_{1}\left(\tilde{k}_{x}\right)$, где $\tilde{k}_{x}$ - вектор обратной решетки, приведенный к ее первой ячейке, $\Delta \omega_{s}$ - ширина зоны. Величина $E_{s}(0)$ нормирована так, что среднее значение $E_{s}$ удовлетворяет условию $\left\langle E_{s}\left(\tilde{k}_{x}\right)\right\rangle=\omega_{s}$. Как уже отмечалось выше, в обшем случае коэффициент скорости включает вероятность перехода $W\left(s^{\prime \prime}, \tilde{\nu}_{q} \mid s^{\prime \prime}-1, \tilde{\nu}_{q}+1\right)$. Поскольку учет уширения уровней зоны вносит дополнительную зависимость от $s^{\prime \prime}$, релаксационная постоянная в обшем случае также включает дополнительную сумму по $s^{\prime \prime}$, причем под знак суммы входит выражение, зависящее от переменной $s^{\prime \prime}$. Вынесем вероятность $W$ из-под знака суммы при $s^{\prime \prime}=s_{0}$, поскольку в этом случае частоту релаксации удается представить в форме (10), что предпочтительно с точки зрения теории переходов [18]. В результате указанных преобразований при $\gamma_{s}^{2} \ll \Delta \omega_{s}^{2}$ получаем, что

$$
\frac{1}{\tau^{(\mathrm{ph})}}=2 \pi \frac{\omega_{s_{0}}}{\Omega} \Psi_{1}\left(v_{0}\right) \sum_{q, \tilde{k}_{x}}\left|I_{q}\right|^{2} \varphi\left(\beta_{1} \omega_{q}\right) \delta\left(E_{s_{0}}\left(\tilde{k}_{x}\right)-E_{s_{0}-1}\left(\tilde{k}_{x}\right)-\omega_{q}\right) .
$$

Дальнейший расчет $1 / \tau^{(\mathrm{ph})}$ выполним, ограничиваясь рассмотрением межзонных переходов с минимальной энергией $\zeta_{E}=\min \left\{E_{s_{0}}\left(\tilde{k}_{x}\right)-E_{s_{0}-1}\left(\tilde{k}_{x}\right)\right\}$. Ограничение такими переходами позволяет учитьвать только предельные значения энергий зон, тогда как в общем случае при изучении всех возможных внутризонных и межзонных переходов необходимо использовать более детальное описание энергетических зон, включая введение функции вида $f_{1}\left(\tilde{k}_{x}\right)$ [19]. Более того при расчете (14) будем предполагать, что $\left(\omega_{q}\right)_{\max } \geqslant \zeta_{E}$ и, следовательно, возможны процессы однофононного рассеяния КЧ. Грубая оценка $\zeta_{E}$, выполненная в соответствии с методами работ $[19,20]$, показала, что условие реализации однофононных процессов выполняется в ряде случаев [21, 22]. В результате находим, что

$$
\frac{1}{\tau^{(\mathrm{ph})}}=\frac{\Omega}{\widetilde{\omega}} \mathcal{G} \frac{\widetilde{\omega}}{\zeta_{E}} \varphi\left(\beta_{1} \zeta_{E}\right) \frac{1}{\tau_{1}\left(v_{0}\right)} .
$$

Полученная величина представляет собой обобшение релаксационной постоянной (13) на случай зон конечной ширины и, естественно, включает дополнительный параметр $\zeta_{E}$.

Наконец, вычислим величину $\Psi_{1}\left(v_{0}\right)(12 \sigma)$, определяющую вклад упругого рассеяния в мнимую часть массового оператора. В случае больших скоростей КЧ $v_{0}>v_{a}=$ $Z_{1}^{2 / 3} e^{2}$, где $Z_{1}$ - заряд быстрой частицы, достаточно рассмотреть малоугловое рассеяния КЧ в плоскости $(y, z)$, когда $\Delta p=2 p_{0} \sin (\vartheta / 2) \simeq p_{0} \vartheta$. Здесь $\vartheta$ - угол рассеяния, $p_{0}=m v_{0}-$ продольный импульс КЧ. Принимая во внимание тот факт, что движение КЧ в плоскости $(y, z)$ является свободным, вычислим матричный элемент оператора $V(r)$, используя в качестве волновых функций плоские волны. Затем подставим полученное выражение в (12б) и проинтегрируем по угловой переменной. Таким образом, величину $\Psi_{1}\left(v_{0}\right)$ удается выразить через минимальный угол ядерного рассеяния $\theta^{(n)}\left(v_{0}\right)$, ниже которого сечение рассеяния подавляется экранировкой [23]. Получаем, что

$$
(2 \pi)^{-3} \Psi_{1}\left(v_{0}\right)=\left(\frac{\pi}{8}\right)^{1 / 2} N^{2}(a l \bar{d})^{2} \theta^{(n)}\left(v_{0}\right) .
$$


Формулы (13), (15), (16) дают окончательное выражение для частоты релаксации поперечной энергии в условиях фононного рассеяния.

Вычислим частоту релаксации за счет электронного рассеяния, сопровождаемого переходами КЧ между ближайшими энергетическими уровнями $s \rightarrow s \pm 1$. Найдем затухание $\gamma_{s}^{(\mathrm{e})}=\left(1 / \tau^{(\mathrm{e})}\right)\left(\omega_{s} / \omega_{s_{0}}\right)$, предполагая, что хорошо каналированные частицы взаимодействуют только с валентными электронами кристалла [24]. В работе [25], где дан вывод уравнения Фоккера-Планка для функции Грина (4) в условиях электронного рассеяния, было показано, что общий вид частоты релаксации может быть записан в форме

$$
\frac{1}{\tau^{(\mathrm{e})}}=2 \sum_{\mathbf{k}_{1} \mathbf{k}_{2}} P_{\mathbf{k}_{1} \mathbf{k}_{2}} I_{\widetilde{\mathbf{k}}_{1} \widetilde{\mathbf{k}}_{2}} \delta\left(\widetilde{\omega}_{k_{1} k_{2}}-\Omega\right)
$$

где

$$
\begin{aligned}
P_{\mathbf{k}_{1} \mathbf{k}_{2}} & =\frac{\pi}{m_{\mathrm{e}}}\left|\widetilde{V}_{\mathbf{k}_{1}-\mathbf{k}_{2}}\right|^{2}\left\{\left(k_{1}\right)_{x}-\left(k_{2}\right)_{x}\right\}^{2} \tilde{n}_{\mathbf{k}_{1}}\left(1-\tilde{n}_{\mathbf{k}_{2}}\right) \\
I_{\widetilde{\mathbf{k}}_{1} \widetilde{\mathbf{k}}_{2}} & =\frac{2 \pi}{\bar{d}} \sum_{\Delta} \delta\left(k_{y}-\Delta_{y}\right) \delta\left(T_{\tilde{k}_{1 z}}-T_{\tilde{k}_{2 z}}\right) .
\end{aligned}
$$

Здесь $\widetilde{V}_{\mathbf{k}}-$ фурье-компонента кулоновского потенциала, $T_{k}-$ кинетическая энергия электрона, $\widetilde{\omega}_{k k^{\prime}}=T_{k}-T_{k^{\prime}}, \quad \Delta=\left(\Delta_{y}, \Delta_{z}\right)$ - двумерный импульс передачи, $\widetilde{\mathbf{k}}=$ $\left(k_{y}, k_{z}\right), m_{\mathrm{e}}-$ масса электрона, $\tilde{n}_{\mathbf{k}}-$ функция распределения электронов.

В случае легких частиц с энергией порядка 1 МэВ скорость $v_{0}$ всегда превышает скорость Ферми $v_{\mathrm{F}}$. Поэтому при вычислении суммы (17) ограничимся случаем $v_{0}>v_{\mathrm{F}}$ и, кроме того, пренебрежем температурным "размазыванием" распределения $\tilde{n}_{\mathbf{k}}$, рассматривая электронный газ в основном состоянии. В этих условиях аппроксимация $\widetilde{\omega}_{k_{1} k_{2}}$ очевидна [26], так же как аппроксимация $P_{\mathbf{k}_{1} \mathbf{k}_{2}}$, если учесть, что кулоновский потенциал $\widetilde{V}_{\mathbf{k}}$ не экранирован.

В работе [27] представлены три типа диаграмм в $\mathbf{k}$-пространстве, описывающих различные варианты рассеяния частицы на свободных электронах с учетом принципа Паули, как того требует условие $P_{\mathbf{k}_{1} \mathbf{k}_{2}} \sim \tilde{n}_{\mathbf{k}_{1}}\left(1-\tilde{n}_{\mathbf{k}_{2}}\right)$. При вычислении (17) будем использовать диаграмму работы [27] для случая $v_{0}>v_{\mathrm{F}}$, включая пределы интегрирования и области разрешенных углов для $\mathbf{k}_{1}$ и $\mathbf{k}_{1}-\mathbf{k}_{2}$. Если считать, что весь импульс $\Delta_{y}$ и энергия $Q\left(\Delta_{y}\right)$ передаются одному электрону, то [26] $Q\left(\Delta_{y}\right)=\Delta_{y}^{2} /\left(2 m_{\mathrm{e}}\right)$ и при расчете (17) удобнее отнести сечение рассеяния $d \sigma\left(\Delta_{y}\right)$ не к элементу телесного угла, а к элементу $d Q\left(\Delta_{y}\right)$. Тогда в условиях больших скоростей $[26]$ имеем

$$
d \sigma\left(\Delta_{y}\right)=2 \pi Z_{1}^{2} e^{4} \frac{1}{m_{\mathrm{e}} v_{0}} N \widetilde{Z}_{2} \frac{d Q\left(\Delta_{y}\right)}{Q^{2}\left(\Delta_{y}\right)}
$$

где $N \widetilde{Z}_{2}$ - плотность валентных электронов. Выполнив суммирование в (17) с учетом 
сделанных выше замечаний, получаем, что

$$
\begin{gathered}
\frac{1}{\tau^{(\mathrm{e})}}=\Omega^{2}\left(\frac{l}{v_{\mathrm{F}}}\right)^{2} \frac{1}{\tau_{2}\left(v_{0}\right)}, \\
\frac{1}{\tau_{2}\left(v_{0}\right)}=\frac{2 a_{\mathrm{TF}}^{2}}{\Omega} \int d \sigma\left(\Delta_{y}\right) \pi\left(\Delta_{y}, \Omega\right) Q\left(\Delta_{y}\right) .
\end{gathered}
$$

Поляризационный оператор в (18б)

$$
\begin{aligned}
\pi\left(\Delta_{y}, \Omega\right)= & \frac{q_{\mathrm{TF}}^{2}}{4 \pi}\left\{\frac{1}{2}+\frac{k_{\mathrm{F}}}{4 \Delta_{y}}\left[\left(1-\frac{\left(\Omega-\Delta_{y}^{2} / 2 m_{\mathrm{e}}\right)^{2}}{\Delta_{y}^{2} v_{\mathrm{F}}^{2}}\right) \ln \left|\frac{\Omega-\Delta_{y} v_{\mathrm{F}}-\Delta_{y}^{2} / 2 m_{\mathrm{e}}}{\Omega+\Delta_{y} v_{\mathrm{F}}-\Delta_{y}^{2} / 2 m_{\mathrm{e}}}\right|+\right.\right. \\
& \left.\left.+\left(1-\frac{\left(\Omega+\Delta_{y}^{2} / 2 m_{\mathrm{e}}\right)^{2}}{\Delta_{y}^{2} v_{\mathrm{F}}^{2}}\right) \ln \left|\frac{\Omega+\Delta_{y} v_{\mathrm{F}}+\Delta_{y}^{2} / 2 m_{\mathrm{e}}}{\Omega-\Delta_{y} v_{\mathrm{F}}+\Delta_{y}^{2} / 2 m_{\mathrm{e}}}\right|\right]\right\}
\end{aligned}
$$

обеспечивает учет пространственно-временных корреляций в процессах рассеяния. Величина $q_{\mathrm{TF}}$ в формуле (19) является волновым вектором модели Томаса-Ферми.

Вследствие сложного вида (19) аналитическое выражение для $1 / \tau_{2}(18 б)$, которое получается в результате интегрирования по $d Q\left(\Delta_{y}\right)$, является громоздким. Его можно упростить, если ограничиться учетом столкновений КЧ с одночастичными возбуждениями за счет близкой части кулоновского взаимодействия. Такие столкновения сопровождаются большими передачами импульса $\Delta_{y} \sim\left(\omega_{\mathrm{pl}} / v_{\mathrm{F}}\right), v_{\mathrm{F}} \Delta_{y} \gg \Omega$, где $\omega_{\mathrm{pl}}-$ энергия плазмона. В этом случае поляризационный оператор (19) заменяется в (18б) его квазистатическим пределом $\pi\left(\Delta_{y}, 0\right)$.

Однако значительного упрошения (18б) можно достигнуть в еще более грубом приближении. Отметим некоторое обстоятельство, которое выяснилось при расчете энергии связи протона с металлом [28], а также обменной энергии системы протон-металл [29]. Сравнение аналитического результата с данными численного интегрирования показало [28, 29], что в подынтегральной функции вида (18б) поляризационный оператор можно аппроксимировать его значением в некоторой точке из интервала интегрирования $\left(\omega_{\mathrm{pl}} / v_{\mathrm{F}}\right) \leqslant \Delta_{y} \leqslant 2 m_{\mathrm{e}} v_{0}$, а именно, значением $\pi(0,0) / 1.6$. Используя это приближение, получаем, что

$$
\begin{gathered}
\frac{1}{\tau_{2}\left(v_{0}\right)}=\chi_{2} \Psi_{2}\left(v_{0}\right), \quad \chi_{2}=\frac{5}{8} \frac{1}{\Omega}\left(Z_{1} e^{2}\right)^{2} N \widetilde{Z}_{2} \bar{d} \\
\Psi_{2}\left(v_{0}\right)=\frac{1}{10} \ln \left(\frac{2 m_{\mathrm{e}} v_{0} v_{\mathrm{F}}}{\omega_{\mathrm{pl}}}\right) \theta^{(\mathrm{e})}\left(v_{0}\right),
\end{gathered}
$$

где $\theta^{(\mathrm{e})}\left(v_{0}\right)$ - минимальный угол электронного рассеяния [23]. Фактически найдено выражение для (18б) в случае резерфордовского рассеяния, когда процесс столкновения рассматривается без учета влияния экранировки [30]. 


\section{4. ЗАКЛЮЧЕНИЕ}

Работы, в которых исследовалась скорость выхода частиц из режима каналирования, в большинстве своем основаны на методах механики, не позволяющих последовательно получать аналитические выражения для кинетических коэффициентов. Расчет в них ограничивался численным интегрированием уравнений для конкретных случаев (см. в качестве примера численный анализ коэффициента диффузии в пространстве поперечной энергии $[31,32])$. Что же касается энергетических потерь, то расчет потерь [33] сводился к численному интегрированию эффективного сечения торможения.

Другое направление теории каналирования представлено в работах $[11,12,14,25]$. Оно основано на методах неравновесной статистической термодинамики. В частности, в работе [11] для системы, включаюшей пучок быстрых частиш и кристалл, методом неравновесного статистического оператора дан вывод коэффициента скорости деканалирования. В отличие от коэффициента скорости в теории абсолютных скоростей [34], обобщенное выражение для кинетического коэффициента [11] (см. формулу (1)) описывает скорость процесса в широком интервале термодинамических условий, в том числе в сильно неравновесной системе. Такой системой является изучаемая нами система в условиях каналирования быстрых частиц. Рассматривая КЧ как “горячие” атомы, мы установили наличие в кинетическом коэффишиенте (8) дополнительного множителя функции $\nu\left(F_{21}\right)$, зависящей от поперечной квазитемпературы направленного пучка. Эта функция не имеет аналога ни в классической кинетической теории, ни в аррениусовской кинетике, поскольку ее появление полностью связано с отрывом термодинамических параметров быстрых частиц от соответствующих параметров термостата.

В исследованиях эффекта каналирования, в которых применялись методы квантовой теории, фактически использованы "гибридные" подходы в том смысле, что быстрые частищы рассматривались как классическая подсистема, а термостат - как квантовая. Типичным примером может служить работа [35]. В отличие от этих работ в нашей теории переход $\{2\} \rightarrow\{3\}$ происходит не поверх потенциального барьера, а путем квантово-механического резонанса через возбужденное состояние $s_{0}$. В свою очередь, достижение частицей уровня $\omega_{s_{0}}$ является результатом случайных блужданий между уровнями $\omega_{s}$ и также рассматривается в рамках квантовой теории. Такая формулировка элементарного акта деканалирования находит свое отражение в структуре коэффициента скорости (8), которая включает следующие качественно новые элементы:

- матричный элемент резонансного перехода (9). При $k_{c} \gg 1$ с точностью до постоянного множителя порядка единицы матричный элемент перехода $\{2\} \rightarrow\{3\}$ равен матричному элементу туннелирования [36] между основными состояниями симметричной двухъячеечной ямы, состоящей из двух гармонических ям;

- частоту релаксации поперечной энергии за счет одноффононного рассеяния КЧ (15), выраженную через основные параметры теории. Подчеркнем, что при выводе (15) рассматривалось не движение быстрой частицы в “замороженной” решетке, как это делается в теории Линдхарда [1], а взаимодействие частицы с фононами. Учет колебательной моды решетки приводит к тому, что в частоту релаксации (15) вошла новая функция 
$\mathcal{G}\left(\widetilde{\omega} / \zeta_{E}\right)$, явньй вид которой (13б) определяется законом дисперсии волн решетки (11);

- частоту релаксации поперечной энергии за счет электронного рассеяния КЧ (18a), (20), выраженную через основные параметры теории. В расчете учитьвалось рассеяние быстрых частиц на одночастичных возбуждениях электронного газа в основном состоянии. Релаксационная постоянная выражена с использованием стандартного сечения рассеяния в случае больших скоростей частиц и поляризационного оператора (19). Такая формулировка частоты релаксации находится в прямой связи с соотношениями теории необратимых процессов Ван Хова [37].

ПРИЛОЖЕНИЕ

Рассмотрим плотность распределения частищ в состоянии квазиравновесия, т.е. на глубине проникновения частиц порядка длины когерентности. Как известно [38], на столь малых глубинах проникновения неупругое рассеяние частиц неэффективно и плотность распределения удовлетворяет уравнению Лиувилля

$$
\frac{\partial}{\partial x} g(x, \theta, z)+\theta \frac{\partial}{\partial x} g(x, \theta, z)-\frac{1}{2} \frac{\partial \tilde{u}}{\partial x} \frac{\partial}{\partial \theta} g(x, \theta, z)=0,
$$

где $\theta=v_{x} / v_{0}, \tilde{u}(x)=U_{2}(x) / U_{0}, 0 \leqslant x<l$. В случае гауссовской расходимости пучка известное начальное условие для плотности распределения запишется в виде

$$
\left.g(x, \theta, z)\right|_{z=0}=\left[2 \pi\left(\delta \theta_{0}^{2}\right)\right]^{-1 / 2} \exp \left[-\frac{\left(\theta-\theta_{0}\right)^{2}}{2 \delta \theta_{0}^{2}}\right]
$$

где $\left[\delta \theta_{0}^{2}\right]^{1 / 2}$ - угловая расходимость пучка в центре канала. Введем также граничное условие

$$
g(0, \theta, z)=g(2 l, \theta, z),
$$

которое отражает периодичность расположения кристаллографических плоскостей, вдоль которых движутся частицы. В отсутствие неупругого рассеяния, когда поперечная энергия частиц сохраняется, функция распределения также является периодической функцией в $z$-направлении, т.е. $g(x, \theta, z)=g(x, \theta, z+\Lambda)$, так что, усредняя $g(x, \theta, z)$ по глубине, мы получаем ее стационарное значение

$$
g(x, \theta)=\lim _{\Lambda \rightarrow \infty} \frac{1}{\Lambda} \int_{0}^{\Lambda} d z g(x, \theta, z)
$$

Переходя в $\varepsilon_{\perp}$-представление [3], находим, что

$$
G\left(\varepsilon_{\perp}\right)=\int d x g(x, \theta(x)) \frac{1}{\varkappa\left(\varepsilon_{\perp}\right)}\left\{\frac{1}{m}\left(\varepsilon_{\perp}-U_{2}(x)\right)\right\}^{-1 / 2},
$$


где

$$
\begin{aligned}
\theta(x) & =\frac{1}{v_{0}}\left\{\frac{1}{m}\left(\varepsilon_{\perp}-U_{2}(x)\right)\right\}^{1 / 2}, \\
\varkappa\left(\varepsilon_{\perp}\right) & =\frac{1}{2 m} \int d x\left\{\frac{1}{m}\left(\varepsilon_{\perp}-U_{2}(x)\right)\right\}^{-1 / 2} .
\end{aligned}
$$

Величина $G\left(\varepsilon_{\perp}\right)$ имеет смысл плотности состояний КЧ с поперечной энергией, лежащей в интервале значений от $\varepsilon_{\perp}$ до $\varepsilon_{\perp}+d \varepsilon_{\perp}$.

Большинство аналитических расчетов в теории каналирования выполнено с использованием модельных представлений, согласно которым на глубине порядка длины когерентности устанавливается статистическое квазиравновесие в подсистеме частиц направленного пучка. Это обосновывается тем, что на указанной глубине в уравнении движения для матрицы плотности КЧ затухают амплитуды вероятности, но сохраняются ее диагональные матричные элементы, характеризуюшие заселенность отдельных уровней поперечной энергии. Редкие попытки сформулировать квазиравновесные распределения частиц до самого последнего времени делались только в рамках микроканонического рассмотрения, поскольку в теории каналирования отсутствовал такой важньй параметр, как модуль канонического распределения, которым является поперечная квазитемпература частиц. Поэтому вычисление плотности числа состояний направленного пучка частиц представляет интерес для всех направлений теории каналирования. $\mathrm{K}$ сожалению, не удается найти аналитическое выражение для функции $G\left(\varepsilon_{\perp}\right)$. Результат численного интегрирования уравнения (П.2), где функция $g(x, \theta, z)$ удовлетворяет уравнению Лиувилля (П.1), представлен кружочками на рис. 1 в случае протонов с энергией $0.5 \mathrm{MэB,} \mathrm{движушихся} \mathrm{в} \mathrm{канале} \mathrm{(110)} \mathrm{монокристалла} \mathrm{кремния.} \mathrm{Предполага-}$ лось, что $\theta_{0}=0.3 \psi_{\mathrm{c}}, \psi_{\mathrm{c}}-$ критический угол каналирования [1], угловая расходимость равна $0.1 \psi_{\mathrm{c}}$.

\section{Список литературы}

[1] Й. Линдхард. УФН. 1969. Т. 99. № 2. С. 249.

[2] Ю. А. Каилев, Н. М. Садыков. ТМФ. 1983. Т. 54. № 3. С. 456.

[3] Ю. А. Кашлев, Н. М. Садыћов. ТМФ. 1997. Т. 111. № 3. С. 483.

[4] Ю. А. Кашлев, Н. М. Садыков. ФММ. 1990. № 9. С. 19.

[5] L. T. Chadderton. Rad. Eff. 1975. V. 27. № 1. P. 12.

[6] F. Abel, C. Cohen. Phys. Rev. B. 1975. V. 12. № 11. P. 4617.

[7] E. Kuhrt, F. Taubner. Phys. Stat. Sol. 1980. V. 61. № 2. P. 513.

[8] M. Kato. Nucl. Instr. Methods. 1989. V. 39. P. 30.

[9] L. Wielunski. Nucl. Instr. Methods. 1980. V. 168. P. 323.

[10] G. J. Kutcher, M. H. Mittleman. Phys. Rev. B. 1975. V. 11. № 1. P. 125.

[11] Д. Н. Зубарев, Ю. А. Кашлев. ТМФ. 1976. Т. 29. № 3. С. 376.

[12] Ю. А. Кашлев. ТМФ. 1977. Т. 30. № 1. С. 82.

[13] Ю. М. Каган, Ю. В. Кононец. ЖЭТФ. 1974. Т. 66. № 5. С. 1693.

[14] Ю. А. Кашлев. ТМФ. 1995. Т. 102. № 1. С. 106.

[15] R. Kubo. Phys. Rev. 1952. V. 86. № 6. P. 929. 
[16] R. Kubo, Y. Toyozawa. Progr. Theor. Phys. 1955. V. 13. № 2. P. 160.

[17] I. V. Andersen, L. C. Feldman. Phys. Rev. B. 1970. V. 1. № 1. P. 2063.

[18] J. A. Sussmann. Ann. Phys. 1971. V. 6. P. 135.

[19] D. Lepski. Phys. Stat. Sol. 1969. V. 35. № 2. P. 697.

[20] P. Gosar. Nuovo Cimento. 1964. V. 31. № 10. P. 781.

[21] F. Abel, G. Amsel, M. Bruneaux, C. Cohen, A.L. Hoir. Phys. Rev. B. 1976. V. 13. № 3. P. 993.

[22] S. Datz, C.D. Moak, T.S. Noggle, B.R. Appleton, H. O. Lutz. Phys. Rev. 1969. V. 179. № 2. P. 315 .

[23] Д. Бом. Квантовая теория. М.: ГИФМЛ, 1961. С.639.

[24] S. Datz. J. Phys. 1979. V. 40. P. 1.

[25] Ю. А. Кашлев. ТМФ. 1979. Т. 41. № 1. С. 89.

[26] Л. Ландау, Е. Лифиич. Квантовая механика. М.-Л.: ГИТТЛ, 1948. С. 567.

[27] S. P. Ali, D. F. Gallaher. J. Phys. C: Solid State Phys. 1974. V. 7. P. 2434.

[28] Г. С. Соловьев. ЖЭТФ. 1975. Т. 68. № 4. С. 1324.

[29] Г. С. Соловьев. Вопросы теории электронной структуры и энергии взаимодействия водорода в металлах: Автореф. дисс. на соиск. уч. ст. канд. физ.-мат. наук. Киев: ИТФ, 1976.

[30] Д. Динс, Д. Винйард. Радиационные эффекты в твердых телах. М.: ИИЛ, 1960. С.17.

[31] T. Waho, Y. H. Ohtsuki. Rad. Eff. 1976. V. 27. № 2. P. 151.

[32] T. Waho. Phys. Rev. B. 1976. V. 14. № 6. P. 4830.

[33] J. D. Melvin, T. A. Tombrello. Rad. Eff. 1975. V. 26. № 1. P. 113.

[34] I. Prigogine, T. A. Bak. J. Chem. Phys. 1959. V. 31. P. 1368.

[35] M. Rahman. Phys. Rev. B. 1995. V. 52. № 5. P. 3125.

[36] I. Svare. Physica. 1986. V. 141. P. 271.

[37] Van Hove. Phys. Rev. 1957. V. 106. № 4. P. 874.

[38] J. A. Ellison. Phys. Rev. B. 1978. V. 18. № 11. P. 5948.

Поступила в редакцию 4.III.1998 г. 